\title{
Eficacia del Bacillus sphaericus 2362 en el control de larvas de Anopheles pseudopunctipennis (Theobald, 1901) y Culex quinquefasciatus (Say, 1823) en bioensayos de laboratorio
}

\author{
Efficacy of Bacillus sphaericus 2362 in the control larvae of Anopheles \\ pseudopunctipennis (Theobald, 1901) and \\ Culex quinquefasciatus (Say, 1823) in laboratory bioassays
}

\author{
Diana Nongrados', Julia Castro', Carlos Mariños ${ }^{1}$, Alberto Laguna ${ }^{2}$ y Roxana Ríos 2
}

\section{RESUMEN}

Se probó la actividad del Bacillus sphaericus 2362 en formulación líquida contra larvas de Anopheles pseudopunctipennis (Theobald, 1901) y Culex quinquefasciatus (Say, 1823) en bioensayos de laboratorio. Se utilizó agua destilada y agua de criadero, con concentraciones de $1 \times 10^{11}, 1,5 \times 10^{11}, 2,5 \times$ $10^{\prime \prime}, 3,9 \times 10^{\prime \prime}$ esporas $/ \mathrm{mL}$. La población blanco se mantuvo en condiciones de laboratorio, usándose agua y flora nativa de criadero. Se vertieron dosis de $1 \mathrm{~mL}$ en vasos de prueba que contenian 25 larvas del II y $1 \mathrm{II}$ estadio y $150 \mathrm{~mL}$ de agua de criadero o agua destilada respectivamente; se usaron vasos con igual cantidad de agua y larvas como controles para cada concentración, tipo de agua y especie de larva probada. Las pruebas fueron realizadas tres veces para cada concentración, en condiciones de laboratorio. Se realizaron 12 réplicas divididas en 6 para cada especie, usándose un total de 4,800 larvas por especie. Las lecturas de la mortalidad de larvas fueron a las 12, 24, 48 y $72 \mathrm{~h}$ después de añadido Bacillus sphaericus. Se observó la elevada susceptibilidad de Culex quinquefascialus a Bacillus sphaericus 2362, con una mortalidad mayor al $90 \%$ cuando se compararon los grupos tratados y controles (valor de $p=0,031$ y 0,012 para cada tipo de agua respectivamente) a las $48 \mathrm{~h}$ y con una concentración de $1,5 \times 10^{11}$ esporas $/ \mathrm{mL}$.

Se demostró ampliamente que Anopheles pseudopunctipennis no es susceptible a Bacillus sphaericus 2362 en bioensayos de laboratorio y no se encontraron diferencias significativas en los tratamienlos con diferentes lipos de agua (valor de $p>0,05$ ).

Palabras clave: Bacillus sphaericus, Anopheles pseudopunctipennis, Culex quinquefasciatus, control biológico, larvicida.

\section{ABSTRACT}

Bacillus sphaericus 2362 liquid formulation, activity against Anopheles pseudopunctipennis and Culex quinquefasciatus larvae was tested in laboratory bioassays. Breeding place water and distilled water were used with concentrations of $1 \times 10^{\prime \prime}, 1,5 \times 10^{\prime \prime}, 2,5 \times 10^{\prime \prime}, 3,9 \times 10^{\prime \prime}$ spores $/ \mathrm{mL}$. Target population was kept in laboratory conditions by using breeding native water and flora. One $\mathrm{mL}$ doses were added into test cups containing $25 \mathrm{II}$ and III stage larvae and $150 \mathrm{~mL}$ of breeding water or distilled respectively; cups tests with the same amount of water and larvae were used as controls for each concentration, water type and larval specie tested. Testing was made three times for each concentration in laboratory conditions. Twelve replicas were made and divided in six for each species, using a total of 4800 larvae for species. Reading of larvae mortality were at 12, 24, 48, and 72 hours after addition of Bacillus sphaericus. It was observed the high susceptibility of Culex quinquefasciatus to Bacillus sphaericus 2362, with an overall mortality $>90 \%$ as compared with treated and controls ( $p$ value $=0,031$ and 0,012 for each type of water respectively) at 48 hours and with a concentration of 1,5 $\times 10^{11}$ sporesimL.

\footnotetext{
1 Laboratorio de Inmunología Parasıları y Epıdemiologia. Facultad de Ciencias Biológicas, Universidad Nacional Mayor do San Marcos. Av Venezuäla sin. Lima Perú. Emal: dnongrados $\$$ hotmallcom. d19007@ unmsm.edu.pe

2. Olicina General de Epidemiología. MINSA. Grupo ASIS.
} 
It was widely demonstrated that Anopheles pseudopunctipennis is not susceptible to Bacillus sphaericus 2362 in laboratory bioassays and no significant differences were found in treatments with different types of water ( $p$-value $>0,05)$.

Key words: Bacillus sphaericus, Anopheles pseudopunctipennis, Culex quinquefasciatus, biological control, larvicide.

\section{INTRODUCCIÓN}

En el Perú, la malaria producida por Plasmodium vivax y $P$. falciparum se ha convertido en un serio problema de saiud a nivel nacional. Desde su reintroducción, en 1992 a través de la frontera norte y de otras áreas de riesgo como lo son las zonas fronterizas del Perú con Colombia y Brasil y la dispersión del Anopheles darlingi en la Amazonía, la malaria por P. falciparum se ha desplazado al Norte y Oriente del país, estableciendo un patrón ascendente en los departamentos de Loreto, Piura, Tumbes, Lambayeque, Cajamarca, San Martín, Amazonas y La Libertad (OGE MINSA, 1998).

Investigaciones sobre el control de los mosquitos hematófagos han tenido gran influencia en el campo de la salud pública, por el importante papel que cumplen en la transmisión de enfermedades al hombre. En el Perú, se conocen alrededor de 150 especies de mosquitos, de las cuales 43 son del género Anopheles. (Calderón et al., 1995). Entre ellos se encuentran los vectores de malaria, y el resto corresponde al grupo de los Culicíneos que incluyen los géneros Culex, Aedes, Sabethes, vectores de encefalitis equina, dengue y fiebre amarilla silvestre, respectivamente. Han sido incriminados como vectores principales de malaria en el Perú, Anopheles darlingi, An. benarrochi, An. albimanus y An. pseudopunctipennis, éste último de amplia distribución en el país; es altamente doméstico y de marcada preferencia antropofílica. Es considerado el principal vector de malaria en la costa y sierra del Perú (Calderón el al., 1995).
Asimismo, Culex quinquefasciatus y Anopheles pseudopuncripennis son considerados vectores implicados en las epidemias de Encefalitis Equina Venenezolana ocurridas en el Perú (Ministerio de Salud, 1989) en los diferentes nichos ecológicos que presentan las zonas donde ocurrieron las epizootias en nuestro país.

El incremento de enfermedades transmitidas por vectores en los últimos diez años ha creado la necesidad de buscar métodos alternativos para el control vectorial. Los insecticidas de acción residual revolucionaron las estrategias de lucha antivectorial; sin embargo en años recientes su uso indiscriminado ha permitido la aparición y propagación de resistencia a los insecticidas y el surgimiento de formas de comportamiento evasivo en vectores de enfermedades, la contaminación ambiental y el uso creciente de nuevos tipos de insecticidas químicos y con elevados costos ponen de manifiesto que la lucha antivectorial ya no puede basarse exclusivamente de los productos químicos (OMS, 1976). Frente a estos problemas se plantea el método de Control Biológico en la lucha antivectorial como una buena alternativa de control, que consiste fundamentalmente en la manipulación del sistema de regulación natural, en beneficio del hombre (OMS, 1982).

De los métodos de control biológico, el microbiológico ha merecido el mayor apoyo y atención en todo el mundo (OMS, 1982). Las bacterias esporógenas han demostrado ser más específicas en su toxicidad contra organismos blancos que otros insecticidas. Son 
biodegradables, inocuas para el hombre. animales y plantas, además de que su autodispersión asegura su eficacia en sitios inaccesibles y tienen un alto efecto residual que las convierte en biolarvicidas muy económicos al no ser necesaria su aplicación frecuente (Montero et cal., 1991).

Montero et al. (1991) en criaderos temporales y permanentes en la Habana, $\mathrm{Cu}-$ ba, comprobaron la efectividad de Bacillus sphaericus 2362 , en formulación líquida, con dosis de $10 \mathrm{ml} / \mathrm{m}^{2}$, sobre mosquitos Culex quinquefascialus. Anopheles albimanus y Aedes taeniorhynchus, obteniendo $100 \%$ de mortalidad entre las 24 y 72 h postaplicación. Arredondo et al. (1990) probaron 2 formulaciones de Bacillus sphaericus 2362 : una granular y una liquida, sobre larvas de Anopheles albimanus. Culex quinquefasciaus y Culex coronator, en parcelas de campo en Tapachula y Chiapas al sur de México; obtuvieron para larvas de Culex una efectividad mayor al $87 \%$ utilizando $0,125 \mathrm{ml} /$ $\mathrm{m}^{2}$ de formulación líquida y $67 \%$ de efectividad con $0,5 \mathrm{~g} / \mathrm{m}$ ? de formulación granular, después de 12 semanas de tratamiento y una reducción larvaria de $68 \%$ con $2 \mathrm{~g} / \mathrm{m}^{2}$ de formulación granular y $57 \%$ con $2 \mathrm{ml} / \mathrm{m}^{2}$ para larvas de Anopheles después de 18 semanas de tratamiento.

Castro el al. (1996) realizaron aplicaciones con Bacillus sphaericus 2362 en criaderos naturales ubicados en 5 subregiones de Salud obtuvieron una eficiencia de mortalidad larvaria de $96.6 \%$ (La Libertad), 96.9\% (Piura), 89,2\% (Sullana) y $84,6 \%$ (San Martín) de poblaciones de mosquitos de la Familia Culicidae a las 48 h postaplicación con una dosis de $10 \mathrm{ml} /$ $\mathrm{m}^{2}$, mientras que Tumbes mostró valores negativos.

Lacey er al. (1986a) evaluaron en cultivos de arroz en Arkansas, concentraciones de $B a$ cillus sphaericus 2362 contra larvas de mosquitos, demostrando que con dosis de $0.58 \mathrm{y}$
1.17 L/ha se obtuvo una reducción de Anopheles quadrimaculaus de 7 l y $82 \%$ respectivamente a las $48 \mathrm{~h}$ de aplicado. Una semana después de la aplicación, también observaton reducción de la población larvaria.

El objetivo fundamental del presente trabajo fue determinar la susceptibilidad del Bacillus sphaericus 2.362 formulación líquida frente a larvas de Anopheles pseudopunctipennis y Culex quinquefasciatus en condiciones de laboratorio, a través de la reducción larvaria, y determinar si las características físicoquímicas y bacteriológicas del agua influyen en la acción tóxica del bacilo, investigación que aportará al conocimiento de la eficacia del Bacillus sphaericus 2362 en el control de vectores transmisores de enfermedades metaxénicas en nuestro país.

\section{MATERIAL Y MIÉTODOS}

\section{A Identificación de criaderos y man- tenimiento de larvas en el insectario}

Los criaderos se caracterizaron según su permanencia, presencia o no de vegetación y grado de contaminación de las aguas; se identificaron tres criaderos en el área urbana y rural, cercana a la ciudad de Lima, un criadero permanente (Laguná de $500 \mathrm{~m}^{2}$ de área) con larvas de Anopheles pseudopunctipennis, ubicado en el Asentamiento Humano "Nueva Jerusalén" km 20 de la Panamericana Norte, distrito de Carabayllo, cuyas aguas son filtraciones del río Chillón, y 2 criaderos temporales (tanques de cemento para almacenar agua, con un área de $5 \mathrm{~m}^{2}$ ) ubicados en el Fundo Pando, $10 \mathrm{~km}$ de la ciudad de Lima, distrito de San Miguel y en el Asentamiento Humano "Pachacutec" $50 \mathrm{~km}$ de Lima, distrito de Ventanilla con larvas de Culex quinquefasciatus. (Fig. 2).

Las colectas larvarias se realizaron durante los meses, entre julio y diciembre de 1997 y para el análisis de los parámetros físico- 
químicos y bacteriológicos del agua de los criaderos se tomaron muestras de agua en frascos estériles de 1 litro de capacidad que fueron analizadas por el laboratorio de Control Ambiental de la Dirección General de Salud Ambiental (DIGESA) del Ministerio de Salud.

La población blanco fue colectada con bandejas de plástico y separada con cucharones esmaltados de $350 \mathrm{ml}$. Se colectaron todos los estadios larvarios, siendo transportadas al Laboratorio de Inmunología Parasitaria y Epidemiología de la Facultad de Ciencias Biológicas de la Universidad Vacional Mayor de San Marcos. Las muestras colectadas fueron separadas por estadios larvarios I y II y colocadas en bandejas de plástico blanco $(26 \mathrm{~cm}$ de largo x $16 \mathrm{~cm}$ de ancho y $5 \mathrm{~cm}$ de altura) tratando de mantenerlas en condiciones naturales y a temperatura ambiente $\left(27^{\circ} \mathrm{C}\right)$ y humedad relativa de $97 \%$, alimentadas cada 24 horas con 1,5 gramos de alimento para pollos (Purina) pulverizado, durante 7 días previos a los bioensayos para la adaptación a las condiciones de laboratorio.

\section{B Caracterización del biolarvicida, preparación y cuantificación del inóculo}

El biolarvicida utilizado fue Bacillus sphaericus, cepa 2362 en formulación líquida de color gris con nombre comercial GRISELESF, proporcionado por LABIOFAM (Cuba), que contiene esporas y cristales tóxicos, con un título $3 \times 10^{\prime \prime}$ esporas $/ \mathrm{mL}$, con $\mathrm{CL}_{50} 0,090691 \mathrm{mg}$ de esporas/L; un $\mathrm{CL}_{90}$ $0.15058 \mathrm{mg}$ de esporas/L, de aspecto concentrado por fermentación.

Para el aislamiento de las colonias se utilizó como medio de cultivo Agar NYSM (medio caldo nutriente, extracto de levadura y sales) (Yousten y Davidson, 1982) e incubando a $37^{\circ} \mathrm{C}$ durante $24 \mathrm{~h}$. Las colonias aisladas fueron marcadas y sometidas a su identificación morfológica a través de la coloración GRAM y bioquímica diferencial (acidez, gas, acetoína, reducción de nitratos, indol, caldo V-P. hidrólisis de almidón, catalasa, caseína y tirosina). Para el análisis cuantitativo del inóculo, se utilizó el método del recuento estándar en placas, con diluciones seriadas hasta obtener la cuantificación del inóculo por cada dosis de biolarvicida; se realizó diluciones hasta $10^{-11}$ de cada una. El recuento de colonias se hizo por el número de Unidades Formadoras de Colonias (UFC), registrándose como cuenta estándar en placa por mililitro. Se utilizaron 4 diferentes concentraciones de biolarvicida como inóculos en los bioensayos de laboratorio $1 \times 10^{11}, 1,5 \times 10^{11}, 2,5 \times 10^{11}$, $3,9 \times 10^{\prime \prime}$ esporas $/ \mathrm{mL}$ que corresponden a las dosis $50 \mathrm{ml} / \mathrm{L}, 100 \mathrm{ml} / \mathrm{L}, 150 \mathrm{ml} / \mathrm{L}$ y $200 \mathrm{ml} / \mathrm{L}$ respectivamente. Cida inóculo de aplicación fue preparado colocando en tubos de prueba volúmenes de $9,5,9,8.5$ y $8 \mathrm{~mL}$ de agui destilada estéril; se agregó $0.5 \mathrm{~mL}, 1 \mathrm{~mL}, 1,5$ $\mathrm{mL}$ y $2 \mathrm{~mL}$ de biolarvicida puro respectivamente, y se obtuvo un volumen final de $10 \mathrm{ml}$ en todos los tubos con inóculo.

\section{Bioensayos de Laboratorio}

Para el estudio experimental se utilizaron larvas del segundo y tercer estadio de Anopheles preudopunctipennis y Culex quinquefasciatus. En condiciones de laboratorio se hicieron 12 réplicas divididas en 6 por especie. Para los bioensayos se dispusieron de 32 vasos de prueba $(7,5 \mathrm{~cm}$ de alto y $8,5 \mathrm{de}$ diámetro con capacidad de $250 \mathrm{ml}$ ) para cada especie larvaria y se utilizaron dos tipos de agua: destilada y de criadero. En 16 vasos se colocaron $150 \mathrm{ml}$ de agua destilada y en otros 16 vasos $150 \mathrm{ml}$ de agua de criadero, luego se añadieron 25 larvas en cada vaso. Las pruebas se hicieron por triplicado para cada concentración de biolarvicida probada. utilizándose también un vaso controí con la misma densidad larvaria por concentración y por tipo de agua para cada especie larvaria, con un total de 800 larvas por plueba. El procedimiento experimental siguió los lineamientos recomendados por la OMS (WHO, 1985). 
Se tomó la temperatura media del agua destilada y del criadero, así como el pH de las mismas, antes de aplicarse cada una de las concentraciones de biolarvicida; luego se vertió $1 \mathrm{~mL}$ de cada concentración sobre la superficie del agua en cada uno de los recipientes, a los controles sólo se les colocó agua destilada y/o agua de criadero y 25 larvas. Los recuentos de la mortalidad larvaria se realizaron a las $12,24,36,48$ y $72 \mathrm{~h}$ postaplicación. Fueron considuadas larvas muertas aquellas que no reaccionaron cuando se les tocó en la región cefálica; larvas moribundas las que se mantenían por debajo de la superficie del agua o las que no tuvieron la reacción característica de sumergirse.

\section{Análisis de la entomotoxicidad}

El porcentaje de mortalidad larraria por dosis su evidenció con el número total de larvas muertas y moribundas. Cuando la mortalidad larvaria en los vasos control estuvo comprendida entre el 5 - $10 \%$ se comigicron los porcentajes de mortalidad mediante la fórmula de Abbott (WHO, 1985). La toxicidad se reportó como Concentración Letal Media $\mathrm{CL}_{5()}$ y $\mathrm{CL}_{9()}$ según programa de análisis Probit 1.4. Para medir la eficacia del Bacillus sphaericus 2362, se utilizó la prueba paramérrica de análisis de varianza ANOVA y para comprobar la significancia entre tratamientos y medir si la calidad del agua intervino en los resultados obtenidos, se utilizó el método de análisis multivariado (MANOVA) del paquete estadístico SPSSWIN 7.5

\section{RESULTADOS}

Los resultados de los análisis físicoquímicos y microbiológicos de las aguas de los criaderos de culex y Anopheies reportan aguas claras con bajos niveles de polución y se expresan en la Tabla 1

Tabla 1. Características fisicoquímicas y bacteriológicas del agua de los criaderos

\begin{tabular}{|c|c|c|}
\hline Indicadores & $\begin{array}{c}\text { Criadero de An. } \\
\text { pseudopunctipennis }\end{array}$ & $\begin{array}{l}\text { Criadero de } C x \text {. } \\
\text { quinquefasciatus }\end{array}$ \\
\hline$p H$ & 6,9 & 7,8 \\
\hline Temperalura ${ }^{\circ} \mathrm{C}$ & $20^{\circ} \mathrm{C}$ & $21^{\circ} \mathrm{C}$ \\
\hline Turbiedad & 1,79 UNT & 5,6 'JNT \\
\hline Color & 18 Unidad Color & $29, i$ Unidad Color \\
\hline Cloruros & $12 \mathrm{mg} / \mathrm{L} \mathrm{Cl}-1$ & $8 \mathrm{mg} / \mathrm{L} \mathrm{Cl}-1$ \\
\hline Sólidos cotales disueltos & $316 \mathrm{mg} / \mathrm{L}$ & $560 \mathrm{mg} / \mathrm{L}$ \\
\hline Nitritos & $0,03 \mathrm{ppm}$ & $0.15 \mathrm{ppm}$ \\
\hline OD ppm & $1,8 \mathrm{ppm}$ & $0,2 \mathrm{ppm}$ \\
\hline Conduclividad & 407 umhos/cm & 337 umhos/cm \\
\hline NMP colitormes totales & $6,3 \times 105 \mathrm{UFC} / \mathrm{ml}$ & $4,5 \times 104 \mathrm{UFC} / \mathrm{ml}$ \\
\hline NMP coliformes fecales & $1,2 \times 103 \mathrm{UFC} / \mathrm{ml}$ & $3,2 \times 102 \mathrm{UFC} / \mathrm{ml}$ \\
\hline
\end{tabular}


El cultivo de Bacillus sphaericus 2362 en Agar NYSM evidenció colonias cremosas, opacas, de borde irregular, con crecimiento ramificado y abundante. Se observaron bacterias bacilares, y se presentaron esporas esféricas que se sitúan en la parte terminal del esporangio, Gram $(+)$ y pruebas diferenciales [Acidez (-), Gas (-) y Acetoína $(-)$, Caseína (+), Tirosina (-), Hidrólisis del Almidón (-), Reducción de Nitratos (-), Indol $(-)$, Caldo V-P (+) y Catalasa (+)] que caracterizaron a la especie Bacillus sphaericus.

La mortalidad larvaria de Culex quinquefasciatus aumentó en relación directa a la concentración (esporas/mL) en ambos tipos de agua (Fig. 1). Durante el desarrollo experimental, se comprobó la elevada susceptibilidad de larvas del II y III estadio de Culex quinquefasciatus frente a diferentes concentraciones utilizadas de Bacillus sphaericus 2362. También se comprobó por ensayos repetitivos (6 réplicas) que Anopheles pseudopunctipennis no es susceptible a Bacillus sphaericus 2362, porque no se evidenció mortalidad larvaria, con ninguna concentración.

Los porcentajes acumulativos de mortalidad larvaria en agua destilada y agua de criadero de Culex quinquefusciatus frente a Bacillus sphuericus 2362 son presentados en las Tablas 2 y 3 . A las 12 horas de exposición los porcentajes de mortalidad larvária alcanzaron valores menores al $3 \%$ con todas las concentraciones utilizadas y en ambos tipos de agua. A las 24 horas de exposición usando concentraciones mayores a $1.5 \times 10^{11}$ ulic/mL se observó porcentajes acumulativos de mortalidad larvaria mayor al 50\% de la población expuesta en ambos tipos de agua. Los valores máximos de mortalidad se obtuvieron a las 48 horas de exposición, obteniendo porcentajes acumulativos de reducción larvaria del $85,8 \%, 91,1 \%, 96,4 \%$ y

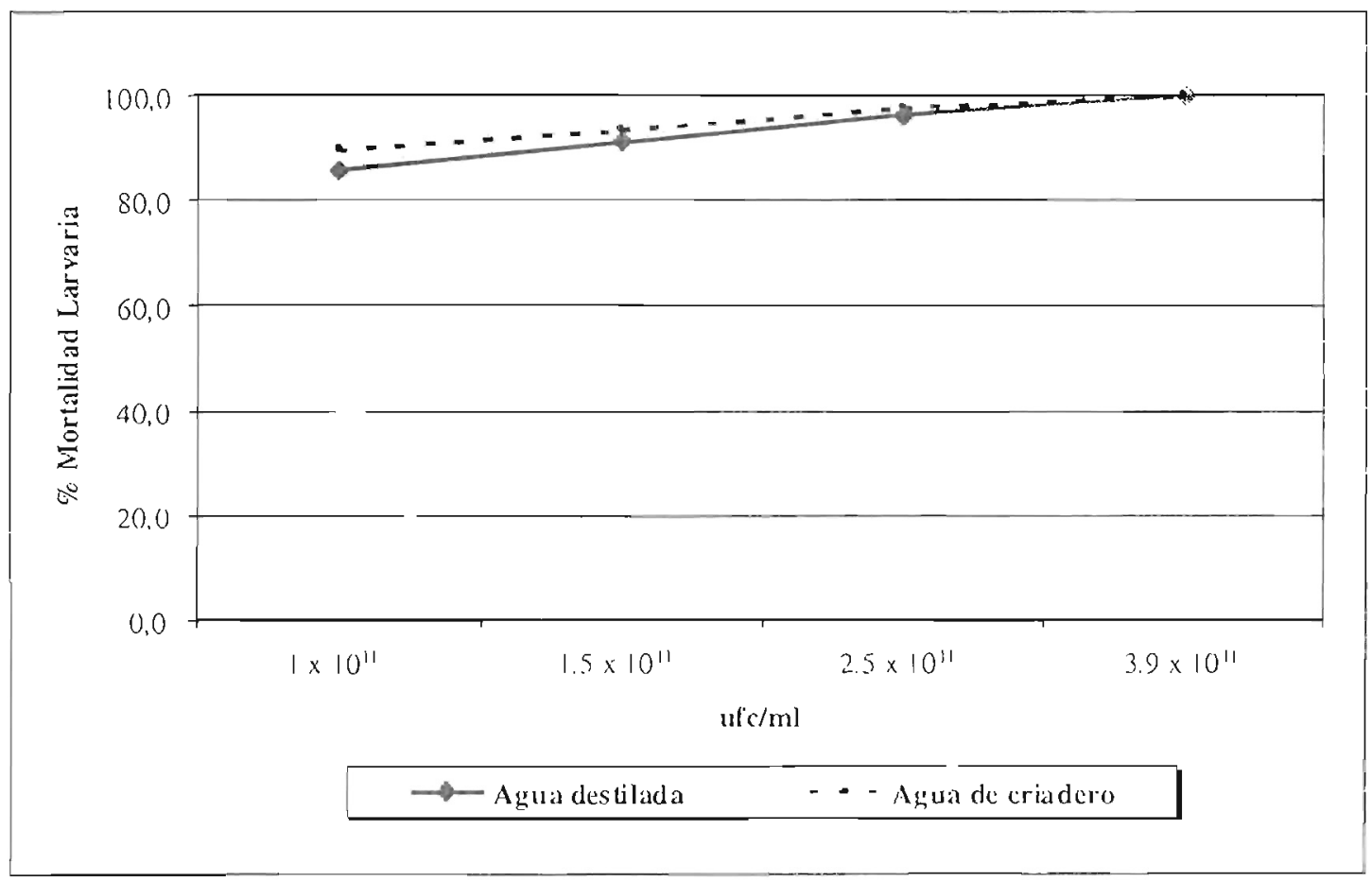

Fig. 1 Curva de Mortalidad Acumulativa de $C x$. quinquefasciatus vs. Concentración de Bacitlus sphaericus 2362 a las 48 horas de exposición 
Tabla 2. Porcentaje acumulativo de mortalidad larvaria de Culex quinquefasciatus y Anopheles pseudopunctipennis con Bacillus sphaericus 2362 en agua destilada

\begin{tabular}{|c|c|c|c|c|c|c|c|c|c|c|}
\hline \multirow{3}{*}{$\begin{array}{c}\text { Concentración } \\
\text { esporas } / \mathrm{ml}\end{array}$} & \multicolumn{10}{|c|}{ Tiempo de exposición } \\
\hline & \multicolumn{2}{|c|}{$12 \mathrm{~h}$} & \multicolumn{2}{|c|}{$24 \mathrm{~h}$} & \multicolumn{2}{|c|}{$36 \mathrm{~h}$} & \multicolumn{2}{|c|}{$48 \mathrm{~h}$} & \multicolumn{2}{|c|}{$72 \mathrm{~h}$} \\
\hline & $C x$ & An. & $C x$ & An. & $\mathrm{Cx}$ & An. & $\mathrm{Cx}$ & An. & $\mathrm{Cx}$ & An. \\
\hline $\begin{array}{l}\text { Control o testigo } \\
\text { (100 larvas II y }(I I)\end{array}$ & 0 & 0 & 0 & 0 & 0 & 0 & 0 & 0 & 0 & 0 \\
\hline $1 \times 1011$ & 0,9 & 0 & 48.4 & 0 & 73,3 & 0 & 85,8 & 0 & 100,0 & 0 \\
\hline $1,5 \times 1011$ & 0,9 & 0 & 58,7 & 0 & 79,6 & 0 & 91,1 & 0 & 100.0 & 0 \\
\hline $2.5 \times 1011$ & 1.3 & 0 & 69.3 & 0 & 84.9 & 0 & 96,4 & 0 & 100.0 & 0 \\
\hline $3,9 \times 1011$ & 1,9 & 0 & 83,1 & 0 & 92.9 & 0 & 100,0 & 0 & 100,0 & 0 \\
\hline $2,2 \times 1011$ & 1,3 & 0 & 64,8 & 0 & 82,6 & 0 & 93,3 & 0 & 100,0 & 0 \\
\hline
\end{tabular}

Tabla 3. Porcentaje acumulativo de mortalidad larvaria de Culex quinquefasciatus y Anopheles pseudopunctipennis con Bacillus sphaericus 2362 en agua de criadero

\begin{tabular}{|c|c|c|c|c|c|c|c|c|c|c|}
\hline \multirow{3}{*}{$\begin{array}{c}\text { Concentración } \\
\text { esporas/ml }\end{array}$} & \multicolumn{10}{|c|}{ Tiempo de exposición } \\
\hline & \multicolumn{2}{|c|}{$12 \mathrm{~h}$} & \multicolumn{2}{|c|}{$24 \mathrm{~h}$} & \multicolumn{2}{|c|}{$36 \mathrm{~h}$} & \multicolumn{2}{|c|}{$48 \mathrm{~h}$} & \multicolumn{2}{|c|}{$72 \mathrm{~h}$} \\
\hline & $C x$ & An. & $C x$ & An. & $C x$ & An. & $C x$ & An. & $C x$ & An. \\
\hline $\begin{array}{l}\text { Conlrol o testigo } \\
(100 \text { larvas }|| \text { y III) }\end{array}$ & 0 & 0 & 0 & 0 & 0 & 0 & 0 & 0 & 0 & 0 \\
\hline $1 \times 1011$ & 1,3 & 0 & 64,9 & 0 & 82,2 & 0 & 89,8 & 0 & 100,0 & 0 \\
\hline $1,5 \times 1011$ & 1,8 & 0 & 69,8 & 0 & 85,3 & 0 & 93,3 & 0 & 100,0 & 0 \\
\hline $2,5 \times 1011$ & 1,8 & 0 & 72,9 & 0 & 89,8 & 0 & 97,8 & 0 & 100,0 & 0 \\
\hline $3,9 \times 1019$ & 2,2 & 0 & 80,4 & 0 & 94,7 & 0 & 100,0 & 0 & 100,0 & 0 \\
\hline $2,2 \times 1011$ & 1,8 & 0 & 72,0 & 0 & 88,0 & 0 & 95,2 & 0 & 100,0 & 0 \\
\hline
\end{tabular}


$100 \%$ en agua destilada y $89,8 \%, 93,3 \%$, $97,8 \%$ y $100 \%$ en agua de criadero con concentraciones de $1 \times 10^{11}, 1,5 \times 10^{11}, 2,5 \times$ $10^{11}, 3,9 \times 10^{11}$ esporas/ml respectivamente. El $100 \%$ de mortalidad Jarvaria se obtuvo a las 72 h de exposición con todas las concentraciones aplicadas en ambos tipos de agua; no se observó mortalidad larvaria en los controles Tablas 2 y 3 .

La prueba paramétrica de ANOVA (Tablas 6 y 7) determinó que la presencia de Bacillus sphaericus 2362 en los vasos de prueba indujo la mortalidad larvaria de Culex quinquefasciatus, y se obtuvo diferencias significativas entre los grupos tratados y control a un nivel de significancia de 0,05 , se obtuvo un valor $p=0,031<0,05$ para agua destilada y $p=0,012<0,05$ para agua de criadero, lo que determina que existen diferencias significativas entre tratamientos. Para probar si la calidad de agua tuvo influencia en los resultados obtenidos se utilizó el Análisis Estadístico de MANOVA (Tablas 8 y 9), donde no se obtuvo diferencias significativas entre los tipos de agua a un nivel de significancia 0.05 . El valor $p=0,789>0,05$, indica que estadísticamente no existen diferencias significativas entre los dos tipos de agua para cualquier nivel de concentración de! biolarvicida. Iguaimente las pruebas individuales para cada nivel de concentración de biolarvicida entre los dos tipos de agua no difieren significativamente $(p=0,847>0,05$, $p=0,905>0,05, p=0,952>0.05, p=0,996>$ $0,05)$ cuando se usa una concentración específica de $1 \times 10^{11}, 1,5 \times 10^{11}, 2,5 \times 10^{\prime \prime}$, $3,9 \times 10^{\prime \prime}$ esporas $/ \mathrm{ml}$ respectivamente.

Lat toxicidad de Bacillus sphaericus 2362 sobre larvas de Culex quinquefusciatus a las $48 \mathrm{~h}$ de exposición presentó valores de $\mathrm{LC}_{5}$ $=0,1750 \mathrm{mg}$ csporas $/ \mathrm{l}$ y $\mathrm{LC}_{\gamma_{0}}=0,7481 \mathrm{mg}$ de esporas/l en agua destilada, asimismo, para agua de criadero los valores de toxicidad fueron de $\mathrm{LC}_{50}=0.1081 \mathrm{mg}$ de esporas $/ \mathrm{L}, \mathrm{y}$ $\mathrm{LC}_{90}=0.5709 \mathrm{mg}$ de esporas $/ \mathrm{L}$, según programa de análisis Probit. Tabias 4 y 5 .

Tabla 4. Estimado de los valores de concentración letal de biolarvicida para agua destilada según Análisis Probit a las $48 \mathrm{~h}$ de exposición

\begin{tabular}{cccc}
\hline \multirow{2}{*}{ Puntos } & $\begin{array}{c}\text { Valor Estimado de } \\
\text { Concentración mg/L }\end{array}$ & \multicolumn{2}{c}{$95 \%$ Limite de Confianza } \\
\cline { 3 - 4 } LC 1,00 & 0,0125 & 0,0000 & Superior \\
LC 5.00 & 0,0271 & 0,0000 & 0,0905 \\
LC 10,00 & 0,0410 & 0,0000 & 0,1376 \\
LC 15,00 & 0,0541 & 0,0000 & 0,1722 \\
LC 50,00 & 0,1750 & 0,0000 & 0,2006 \\
LC 85,00 & 0,5666 & 0,0341 & 0,3880 \\
LC 90,00 & 0,7481 & 0,2067 & 0,8929 \\
LC 95,00 & 1,1292 & 0,7061 & 1,4669 \\
LC 99,00 & 2,4444 & 1,3267 & 12,9543 \\
\hline
\end{tabular}

$\mathrm{LC}_{6 i}=0,1750 \mathrm{ng}$ esporas $/ \mathrm{L}$

$\mathrm{LC}_{\mathrm{x}(\mathrm{x})}=0,7481 \mathrm{mg} \operatorname{cspol} \mathrm{as} / \mathrm{I}$ 
Tabla 5. Estimado de los valores de concentración letal de biolarvicida para agua de criadero según Análisis Probit a las $48 \mathrm{~h}$ de exposición

\begin{tabular}{cccc}
\hline \multirow{2}{*}{ Puntos } & Valor Estimado de & \multicolumn{2}{c}{$95 \%$ Limite de Confianza } \\
\cline { 3 - 4 } & Concentración mg/L & Inferior & Superior \\
\hline LC 1,00 & 0,0053 & 0,0000 & 0,0073 \\
LC 5,00 & 0,0128 & 0,0000 & 0,0908 \\
LC 10,00 & 0,0205 & 0,0000 & 0,1154 \\
LC 15,00 & 0,0281 & 0,0000 & 0,1478 \\
LC 50,00 & 0,1081 & 0,0000 & 0,4780 \\
LC 85,00 & 0,4152 & 0,0000 & 0,6729 \\
LC 90,00 & 0,5709 & 0,0347 & 0,9011 \\
LC 95,00 & 0,9152 & 0,3064 & 1,7995 \\
LC 99,00 & 2,2173 & 1,1134 & 4055.3145 \\
\hline
\end{tabular}

$\mathrm{LC}_{30}=0,1081 \mathrm{mg}$ esporas $/ \mathrm{L}$

LC $_{(x)}=0.5709 \mathrm{mg}$ esporas $/ \mathrm{L}$

Tabla 6. Prueba de Significancia para tratamiento en agua destilada usando Única Suma de Cuadrados Análisis de Varianza (ANOVA)

\begin{tabular}{cccccc}
\hline Fuente de Variación & SS & DF & MS & F & Sig of F \\
\hline Término de error & 34,70 & 15,0 & 2,31 & - & - \\
Bloques & 2570,16 & 5,0 & 514,03 & 222,22 & 0,000 \\
Tratamientos & 27,01 & 3,0 & 9,00 & 3,89 & 0,031 \\
\hline (Total) & 2631,88 & 23,0 & 114,43 & - & - \\
\hline
\end{tabular}

Valor crítico (Sig of $F$ ) resultado de $p=0,031<0,05$

$\begin{array}{ll}\text { SS } & =\text { Suma de Cuadrados } \\ \text { DF } & =\text { Grado de Libertad } \\ \text { MS } & =\text { Cuadrado Medio } \\ \text { F } & =\text { F Estimado } \\ \text { Sig of F } & =\text { Prueba de Significancia }\end{array}$ 
Tabla 7. Prueba de Significancia para tratamiento en agua de criadero usando Única Suma de Cuadrados. Análisis de Varianza (ANOVA)

\begin{tabular}{cccccc}
\hline Fuente de Variación & SS & DF & MS & $F$ & Sig of $F$ \\
\hline Término de error & 8,63 & 15,0 & .58 & - & - \\
Bloques & 2688,67 & 5,0 & 537,73 & 934,15 & 0,000 \\
Tratamientos & 8,97 & 3,0 & 2.99 & 5,19 & 0,012 \\
\hline (Total) & 2706,28 & 23,0 & 117,66 & - & - \\
\hline
\end{tabular}

Valor crítico (Sig of $F$ ) resultado de $p=0,012<0,05$

$\begin{array}{ll}\text { SS } & \text { = Suma de Cuadrados } \\ \text { DF } & =\text { Grado de Libertad } \\ \text { MS } & =\text { Cuadrado Medio } \\ \text { F } & =\text { F Estimado } \\ \text { Sig of F } & =\text { Prueba de Significancia }\end{array}$

\section{DISCUSIÓN}

Los valores máximos de mortalidad que se obtuvieron a las 48 horas de exposición con un promedio de $93 \%$ y $95 \%$ de reducción larvaria, en agua destilada y agua de criadero respectivamente, a las concentraciones de 1 $\times 10^{11}, 1,5 \times 10^{11}, 2,5 \times 10^{11}, 3,9 \times 10^{11}$ esporas/ $\mathrm{mL}$, presentan una mediana $2,2 \times 10^{11} \mathrm{ufc} /$ $\mathrm{mL}$. Los altos valores de $\mathrm{LC}_{50}$ y $\mathrm{LC}_{y()}$ hallados para agua destilada y para agua de criadero nos indican mayor presencia de unidades formadoras de colonias (ufc) en las concentraciones utilizadas con respecto al homogenizado, y asimismo pérdida de la capacidad entomocida, debido a que se necesita mayor carga de inóculo en cada concentración para obtener la misma toxicidad. Esto sugiere la existencia de esporas y de células vegetativas en el homogenizado. $B a$ cillus sphaericus tiene que estar completamente esporulado para mantener elevada su capacidad entomocida (Davidson E., 1984a).

Aun así, son apenas superiores a los reportados por Nicolas el al. (1987), quienes utilizando una solución concentrada que contiene $2 \times 10^{10}$ esporas $/ \mathrm{mL}$ de $B$. sphaericus cepa 2362, en bioensayos de aboratorio, obtuvieron un $98 \%$ de mortalidad de Culex quinquefasciatus a las 48 horas de exposición. Similares resultados se aprecia en el trabajo de Mulla el al. (1991), en ensayos de laboratorio utilizando Bacillus sphaericus 2362 (polvo primario) con títulos de $1 \times 10^{11}$ esporas $/ \mathrm{mL}$ contra larvas del cuarto estadio de Culex quinquefasciaus, obtuvieron un $\mathrm{LC}_{\text {*) }}$ de $0.004 \mathrm{mg} / \mathrm{L}$ a las 48 horas postaplicación, que es 22.7 veces mayor al reportado para el homogenizado comercial. La variación en el nivel de actividad puede ser atribuida a diferentes factores tales como la cepa de larva (en las pruebas se usaron cepas silvestres de Culex quinquefasciaius y Anopheles pseudopuctipennis), edad y vigor de la larva y procedimientos usados en los bioensayos, ya que no todos utilizan los mismos protocolos en el momento de evaluar la toxicidad.

Majori et al. (1987) evaluando en bioensayos de laboratorio ia cepa Bacillus sphatericus 2362 (spray seco) contra larvas de Culex quinquefasciatus reportaron un 
Tabla 8. Prueba de Significancia Multivariado para los tratamientos entre los dos tipos de agua Análisis Multivariado (MANOVA)

\begin{tabular}{cccccc}
\hline Nombre de la Prueba & Valores & F & DF & Error DF & Sig of $F$ \\
\hline PILLAIS & 0,19413 & 0,42157 & 4,00 & 7,00 & 0,789 \\
HOTELLINGS & 0,24090 & 0,42157 & 4,00 & 7,00 & 0,789 \\
WLKIS & 0,80587 & 0,42157 & 4,00 & 7,00 & 0,789 \\
\hline ROYS & 0,19413 & - & - & - & - \\
\hline
\end{tabular}

Valor crítico (Sig of F) resultado de $\mathrm{p}=0,789>0,05$

$\mathrm{F} \quad=$ Estadístico de Prucba

DF $\quad=$ Grado de Libertald

Error DF = Grado de Lihertad del Error

Sig of F = Prueba de Significancia

Tabla 9. Prueba de Significancia Univariado entre los dos tipos de agua a un nivel de dosis de biolarvicida utilizados. Análisis Multivariado (MANOVA)

\begin{tabular}{cccccc}
\hline Variable & Hypoth. SS & Error SS & Hypoth. MS & Error MS & $F$ \\
\hline $0.5 x$ & 4,68750 & 1199,74167 & 4,68750 & 119,974167 & 0,03907 \\
$1 \times$ & 1,92000 & 1271,22667 & 1,92000 & 127,122667 & 0,01510 \\
$1.5 x$ & 0,52083 & 1358,38833 & 0,552083 & 135,838833 & 0,00383 \\
$2 x$ & 0,00333 & 1472,81333 & 0,00333 & 147,281333 & 0,00002 \\
\hline
\end{tabular}

Valor crítico (Sig of F) resultado de:

$\mathrm{p}=0,847>0,05, \mathrm{p}=0,905>0,05, \mathrm{p}=0,952>0,05, \mathrm{p}=0,996>0,05$

Hypoth. SS = Suma de Cuadrados de la Hipótesis

ErrorSS = Suma de Cuadrados del Error

Hypoth. MS = Cuadrado Medio de la Hipótesis

Error MS = Cuadrado Medio del Error

$\mathrm{F} \quad=$ Estadístico de Prueba

Sig of $F \quad=$ Prueba de Significancia

$0,5 \mathrm{x} \quad=50 \mathrm{ml} / \mathrm{L}\left(\mathrm{l} \times 10^{11}\right.$ esporas $\left./ \mathrm{ml}\right)$

$1 \mathrm{x} \quad=100 \mathrm{ml} / \mathrm{L}\left(1.5 \times 10^{11} \mathrm{csporas} / \mathrm{ml}\right)$

$1.5 \times \quad=150 \mathrm{ml} / \mathrm{L}\left(2.5 \times 10^{11} \mathrm{csporas} / \mathrm{ml}\right)$

$2 \mathrm{x} \quad=200 \mathrm{ml} / \mathrm{L}\left(3.9 \times 10^{11} \mathrm{csporas} / \mathrm{ml}\right)$ 
$\mathrm{LC}_{90}$ de 0,031 ppm a las 48 de exposición; asimismo, obtuvieron un $\mathrm{LC}_{50}$ de 0,022 ppm y un $\mathrm{LC}_{y_{0}}$ de $0.130 \mathrm{ppm}$ para larvas del tercer $\mathrm{y}$ cuarto estadio de Anopheles gambiae, a las 48 h de exposición. Consoli et al. (1997) evaluaron Bacillus sphaericus 2362 (concentrado emulsionable) contra larvas del tercer y cuarto estadio de Culex quinquefascialus reportaron en condiciones de laboratorio un $\mathrm{LC}_{50}$ de $1,3 \pm 0,6 \mathrm{mg} / \mathrm{L}$ y $\mathrm{LC}_{9()}$ de 5,5 $\pm 1,5 \mathrm{mg} / \mathrm{L}$, después de $48 \mathrm{~h}$ de exposición.

Davidson et al. (1984b) en bioensayos de laboratorio y en parcelas pequeñas en California, confirmaron que Bacillus sphaericus tiene un restringido rango de hospederos, dependiendo de las condiciones del laboratorio; la mayoría de especies de Culex fueron las más susceptibles para este organismo. El comportamiento de Anopheles sp., parece ser variable; aunque pocas especies de Anophelinos han sido usados en los bioensayos. Por ejemplo, An. albimanus, An. stephensi son completamente sensibles, mientras que An. quadrimaculatus es menos sensible.

Según la OMS (1992), las larvas de mosquitos de los géneros Psorophora y Culex son muy susceptibles a Bacillus sphaericus, seguido por los géneros Mansonia, Anopheles y Aedes, en orden decreciente. Las larvas de Culex tienen un nivel de susceptibilidad muy elevado, del mismo orden que su susceptibilidad a los larvicidas químicos de uso corriente. Las larvas de Anopheles, en general, son 10-20 veces más tolerantes a las toxinas de $B a c i$ llus sphaericus que las de Culex; en cambio, las larvas de Aedes aegypti son mil veces más tolerantes. Diferentes autores han observado este perfil larvicidal para Bacillus sphaericus 2362 y otras cepas, como los revisados por Lacey y Undeen (1986b), donde preparaciones de esporas y cristales de Bacillus sphaericus los aplicaron sobre larvas de Aedes aegypti en dosis de 100 a 1000 veces más, que lo requerido por Culex quinquefasciatus. Existen diversos trabajos que demuestran diferentes niveles de sensibilidad de larvas de mosquitos a las diferentes cepas de Bacillus sphaericus, que hacen pensar que la eficacia y eficiencia de Bacillus sphaericus como controlador biológico efectivo dependen de la especie de mosquito, tipo de agua, cepa bacteriana, formulación del insecticida etc. Los trabajos realizados en Brasil, EE. UU. y Australia han demostrado que $B$. sphaericus 2362 , IB y 2297 son igual de efectivos contra Culex quinquefasciatus y Aedes atropalpus, y que la cepa 1593 es tóxica para Aedes nicromaculis. Otros concluyen que Lacillus sphaericus es más activo contra Culex sp. y Anopheles sp. y menos activo contra Aedes sp. (Baumann et al., 1991; Berry et al., 1993; Porter et al., 1993).

La calidad, tipo de agua, pH y luz solar son factores importantes en la determinación de la eficacia del Bacillus sphaericus 2362 para disminuir la densidad poblacional de larvas de mosquitos. En los bioensiayos se utilizó agua destilada y agua de criadero. El agua del criadero de Culex quinquefasciatus se caracterizó por ser clara, con bajos niveles de polución, abundante vidu animal y vegetal. La presencia de sustancias orgánicas aumentan el proceso de ingestión de partículas alimenticias de las larvas, que actúan como fagoestimulantes en cl agua de criadero (Rished y Mulla, 1989). Los resultados obtenidos en la determinación del $\mathrm{LC}_{5()}$ y $\mathrm{LCC}_{\mathrm{in}, \mathrm{3}}$ en los bioensayos con agua de criadero y agua destilada no indican diferencias significativas.

Bacillus sphaericus provee un elevado nivel de control en aguas claras más que en aguas poluidas (Mian y Mulla., 1983). Estos resultados coinciden con nuestras observaciones. Algunos autores reportan que la actividad de Bacillus sphaericus lue adversamente afectada por elevados niveles de polución en el agua. Des Rochers y García (1984) demostraron que Bacillus sphaericus 2362 persiste y permanece tóxico en una variedad de iipos de agua, pero el agua de desaguadero primario $(80 \mathrm{mg} / \mathrm{L}$ de sólidos suspendidos) tiene efectos negativos en la persistencia bacteriana en comparación con el agua de desaguadero secundaria ( $12 \mathrm{mg} / \mathrm{L}$. 


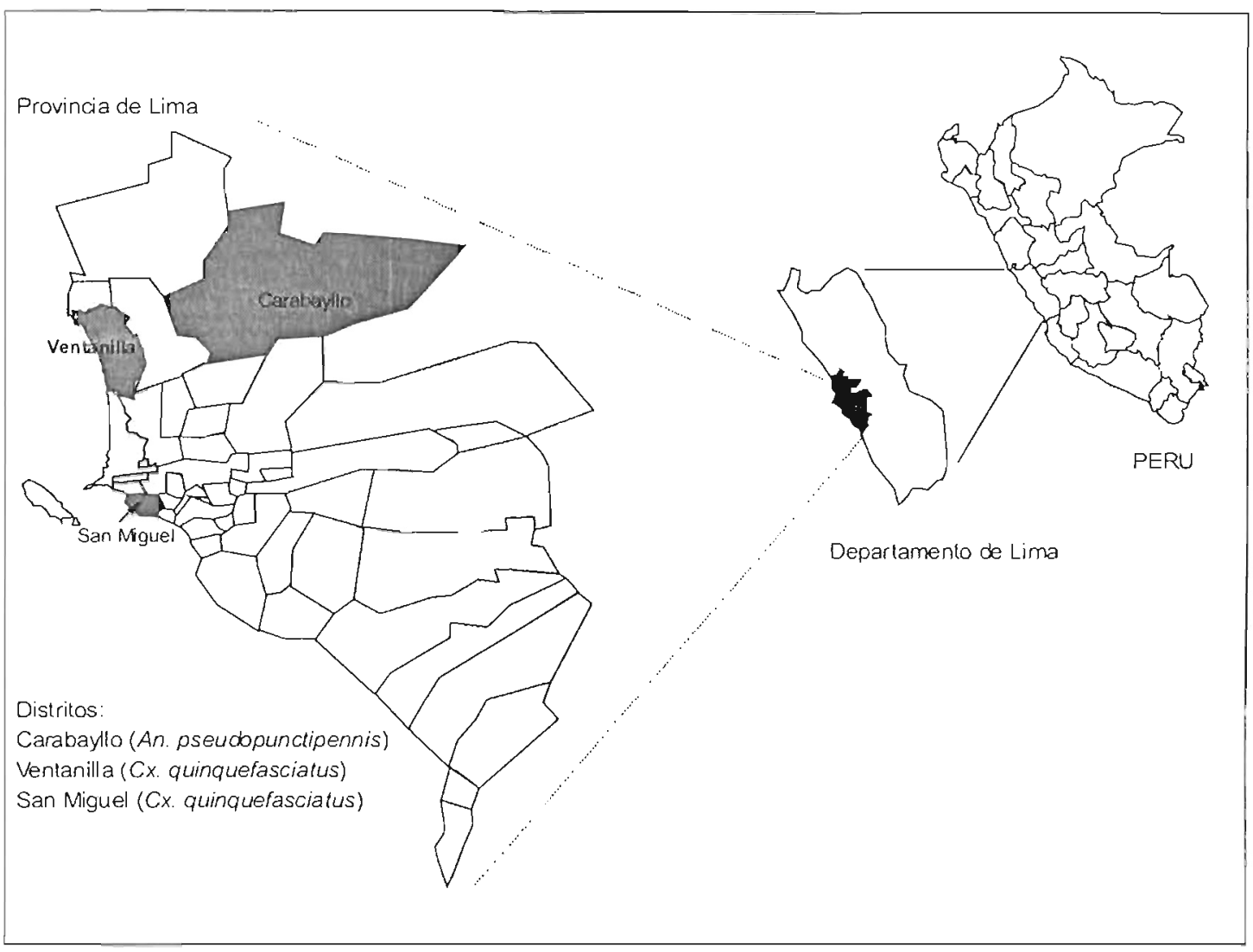

Fig. 2 Ubicación de los criaderos identificados en Lima

de sólidos suspendidos) y agua de lluvia. Jones et al. (1990), en Arkansas, demostraron la eficacia de Bacillus sphaericus cepa 2362 en aguas de acequias de alcantarillado doméstico contra larvas de 2 y 3 estadio de Culex quinquefasciatus después de 48 horas postaplicación, lograron una eficacia de 8093\% de poblaciones larvales.

\section{CONCLUSIONES $Y$ RECOMENDACIONES}

1. La especie Culex quinquefasciatus es susceptible a Bacillus sphaericus 2362, mientras que la especie Anopheles pseudopunctipennis no evidenció susceptibilidad.

2. Concentlaciones mayores de $1,5 \times 10^{11}$ esporas/mL del Bacillus sphaericus 2362 provocaron más del $90 \%$ de reducción larvaria de Culex quinquefasciatus a las 48 horas de exposición.

3. La efectividad de la actividad larvicida de Bacillus sphaericus 2362 contra larvas de mosquitos está en relación con la especie de mosquito y la concentración utilizada.

4. Realizar evaluaciones de campo, en diferentes tipos de criaderos (niveles de polución) y con diferentes especies de anofelinos vectores de malaria.

\section{LITERATURA CITADA}

Arredondo, A., T. Lopez, M. Rodríguez and D. Bown. 1990. Small scale field trials of Bacillus sphaericus (strain 2362) against anopheline and culi- 
cine mosquito larvae in southern Mexico. Journal American Mosquitoes Control Association. 6(2): 300305.

Baumann, P., M. Clark, L. Baumann y A, Broadwell. 1991. Bacillus sphaericus as a Mosquito Pathogen: Properties of the Organism and its Toxin. Microbiology Reviews. 55: $425-436$

Berry, C., J. Hindley, A. Ehrhardt, T. Grounds, I. Souza y E. Davidson. 1993. Genetic determinants of the host range of the Bacillus sphaericus mosquito larvicidal toxins. Journal Bacteriology. 175: 510-518.

Calderón, G., R. Fernández y J. Valle. 1995. Especies de la fauna anophelina, su distribución y algunas consideraciones sobre su abundancia, infectividad e importancia en la transmisión de malaria en el Perú Revista Peruana de Epidemiología. $8(1): 5-53$

Consoli, R., B. De Santos, M. Lamounier, N. Secundino, L. Rabinovitch, C. Silva, R. Alves and N. Carneiro. 1997. Efficacy of a New Formulation of $\mathrm{Ba}$ cillus sphaericus 2362 against Culex quinquefasciatus (Diptera: Culicidae) in Montes Claros, Minas Gerais, Brazil. Memorias do Instituto Oswaldo Cruz, Río de Janeiro. 92(4): 571-573.

Castro, J., García, I. y Neyra, D. 1996. Evaluación del tratamiento con Bacillus sphaericus 2362 en criaderos naturales en zonas de alto riesgo de malaria. Revista Peruana de Epidemiología. 9(2): 18-23.
Davidson, E. W. 1984a. Microbiology, Pathology and Genetics of Bacillus sphaericus: Biological Aspects which are Important to Field Use. Mosquito News. 44(2): 147 - 152.

Davidson, E. W., M. Urbina, J. Payne, M. S. Mulia, H. Darwazeh, H. T. Dulmage and J. A. Correa. 1984b. Fate of Bacillus sphaericus 1593 and 2362 spores used as a Jarvicide in the aquatic environment. Applied and Environmental Microbiology. 47: 125-129.

Des Rochers, B. and R. García. 1984. Evidence for persistence and recycling of Bacillus sphaericus. Mosquito News. 44(2): 160-165.

Jones, J., A. Weathersbee, P. Efird and M. Meisch. 1990. Evaluation of Bacillus sphaericus 2362 against Culex quinquefasciatus in septic ditches Joumal American Mosquitoes Control Association. 6(3): 496-499.

Lacey, L., Heitzman, C. M., Meisch, M. and Biliodeaux, J. 1986a. Becomist Applied Bacillus sphaericus for the Control of Riceland mosquitoes. Journal American Mosquitoes Control Association. 2(4): 548 - 551.

Lacey, L. and A. Undeen. 1986b. Microbial control of black flies and mosquitoes. Annual Reviews Entomology. 31: 265-296.

Majori, G., A. Ali and G. Sabatinelli. 1987 Laboratory and Field Efficacy o Bacillus thuringiensis var. Israelensis and Bacillus sphaericus against Anopheles gambiae S.L. and Culex quinquefasciatus in Ouagadougou. Burkina Faso. Journal imerican Mosquitoes Control Association. 3(1): 20-24 
Ministerio de Salud Perú. 1989. Programa Nacional de Zoonosis. Annales del Seminario Nacional de Zoonosis y Enfermedades de Transmisión. 78$84 \mathrm{pp}$.

Mian, L. S. and M. S. Mulla. 1983. Factors influencing activity of de microbial agent Bacillus sphaericus against mosquito larvae. Bulletin Society Vector Ecology. 8: 128-134.

Montero, G., M. Diaz, A. Marrero y A. Castillo. 1991. Resultados de las Aplicaciones en Pilotaje del biolarvicida Bacillus sphaericus 2362 en criaderos de mosquitos del Municipio Santa Cruz del Norte (Provincia La Habana). Revista Cubana de Medicina Tropical. 43 (1): 39-44.

Mulla, M. S., N. Singh and H. A. Darwazeh. 1991. Delayed Mortality and Morphogenetic Anomalies Induced in Culex quinquefasciatus by the Microbial control Agent Bacillus sphaericus. Journal American Mosquitoes Control Association. 7(3): 412-419.

Nicolas, L and I. Dossou-Yovo. 1987. Differential effects of Bacillus sphaericus strain 2362 on Culex quinquefasciatus and its competitor Culex cinereus in West Africa. Medical and Veterinary Entomology. 1: 23-27.

OGE. Oficina General de Epidemiología (MINSA). 1998. Boletín Epidemiológico. Semana Epidemiológica N. ${ }^{\circ}$ 24.

OMS. 1976. Resistencia de los vectores y reservorios de enfermedades a los plaguicidas. $22^{\circ}$ Informe del Comité de Expertos de la OMS en Insecticidas. Serie de Informes Técnicos N. 585, 91 pp.

OMS. 1982. Lucha Biológica Contra los Vectores de Enfermedades. Sexto Informe del Comité de Expertos de la OMS en Biología de los Vectores y Lucha Antivectorial. Serie de Informes Técnicos N. ${ }^{\circ} 679,39 \mathrm{pp}$.

OMS. 1992. Filariasis Linfática: La enfermedad y los métodos de lucha. Serie de Informes Técnicos $N^{\circ} 821.73$ pp.

Porter, A. G., E. W. Davidson and L. JianWei. 1993. Mosquitocidal Toxins of Bacilli and their Genetic Manipuiation for effective Biological Control of Mosquitoes. Microbiology Reviews. 57(4): 838-861.

Rashed, S. and S. Mulla. 1989. Factors Influencing Ingestion of Particulate Materials by Mosquito Larvae (Diptera: Culicidae). Journal of Medical Entomology. 26(3): $210-$ 216.

Word Health Organization. 1985. Informal Consultation o the development of Bacillus sphaericus as a microbial larvicide. TDR/BCV/Sphaericus/ 85.3 WHO/VBC/l-24.

Yousten, A. and E. Davidson. 1982. Uitra structural Analysis of spores and Parasporal Crystal formed by $B a$ cillus sphaericus 2297. Applied and Environmental Microbiology. 44:1449-1455. 